\title{
Planar maps as labeled mobiles
}

\author{
J. Bouttier, P. Di Francesco and E. Guitter \\ Service de Physique Théorique, CEA/DSM/SPhT \\ Unité de recherche associée au CNRS \\ CEA/Saclay \\ 91191 Gif sur Yvette Cedex, France \\ bouttier@spht.saclay.cea.fr \\ philippe@spht.saclay.cea.fr \\ guitter@spht.saclay.cea.fr
}

Submitted: May 25, 2004; Accepted: Sep 7, 2004; Published: Sep 24, 2004

Mathematics Subject Classifications: Primary 05C30; Secondary 05A15, 05C05, 05C12, 68R05

\begin{abstract}
We extend Schaeffer's bijection between rooted quadrangulations and welllabeled trees to the general case of Eulerian planar maps with prescribed face valences to obtain a bijection with a new class of labeled trees, which we call mobiles. Our bijection covers all the classes of maps previously enumerated by either the two-matrix model used by physicists or by the bijection with blossom trees used by combinatorists. Our bijection reduces the enumeration of maps to that, much simpler, of mobiles and moreover keeps track of the geodesic distance within the initial maps via the mobiles' labels. Generating functions for mobiles are shown to obey systems of algebraic recursion relations.
\end{abstract}

\section{Preliminaries}

\subsection{Introduction}

Maps, like graphs and trees, are fundamental combinatorial objects that appear in many different areas of mathematics, computer science, and theoretical or statistical physics. The groundwork for the enumerative theory of planar maps was laid in the 60's by Tutte [19], who enumerated maps of some particular classes, with some remarkably simple results. For example, the number of rooted planar maps with a given number $n$ of edges is $\frac{2(2 n) ! 3^{n}}{n !(n+2) !}$. For many other classes of maps, no similar closed general formula is known but the corresponding generating functions can be shown to obey algebraic equations. Such results are obtained using, for instance, the formalism developed by Tutte (the so-called recursive decomposition) or the powerful method of matrix integrals developed by physicists [10].

However the proofs involved there are rather of an indirect, non-constructive nature. Of greater mathematical beauty are bijective proofs, where the purpose is to 
establish one-to-one correspondences between classes of maps and some simpler sets whose enumeration is obvious. Such correspondences were first found in the 80's by Cori and Vauquelin [13] and later Arquès [1], but the real development of this subject came with the thesis of Schaeffer [17], who was able to rederive most of Tutte's results through new bijective algorithms cutting maps into trees. It was then realized that these bijections gave insight into some detailed information on the intrinsic geometry of maps such as the geodesic distance, with interesting applications both in statistical physics [6-8] and probability theory $[11,12,16]$.

\subsection{Known results, aim of the paper}

We now introduce a few definitions in order to recall some known results. A planar map is an embedding of a connected graph (which may have loops and/or parallel edges) in the sphere such that the edges are non-intersecting open curves and connected only at their extremities (vertices). The complement of the graph is a disjoint union of simply connected domains (faces). In enumerative theory, two maps differing by an homeomorphism (bicontinuous bijection) of the sphere are considered equivalent, hence the number of maps with a finite number of edges is also finite. Most known results deal with rooted maps, that is maps having a distinguished oriented edge (the root).

In a map, the valence or degree of a face or vertex is the number of its incident edges $^{1}$. Maps with only 4-valent faces are called quadrangulations which are dual to tetravalent maps where all vertices have degree 4 . This class of maps is the one for which bijections are the simplest. There are actually two of them [17]: one between tetravalent maps and so-called blossom trees, the other between quadrangulations and well-labeled trees.

So far, generalizations to wider classes of maps were obtained mainly in terms of blossom trees. A first extension consists of enumerating maps with prescribed vertex valences. The corresponding generating functions are easily derived by considering the general "one-matrix model", or by recursive decomposition [2], while the corresponding bijective proof involves blossom trees with subtle charge constraints [9]. This is drastically simplified in the case of maps with only vertices of even valence, corresponding to even potentials in the matrix model formulation. The corresponding blossom trees [18] then have a simple characterization which makes it possible to re-derive Tutte's compact formulas for the numbers of maps with prescribed (even) vertex valences [20].

More generally, one may as well try to enumerate the bipartite, i.e. vertex-bicolored, maps with prescribed vertex valences of either color, which corresponds to the general "two-matrix model". This problem has many physical applications, including, for instance, the celebrated Ising model on random lattices [3] as well as a whole range of multicritical theories corresponding to minimal models of CFT coupled to 2D quantum gravity [14]. The bijective enumeration via blossom trees was found by Bousquet-Mélou

1 These are counted with multiplicity : more precisely we count the number of incident edge sides (resp. half-edges). 
and Schaeffer [4]. This indeed extends the previous case, as arbitrary maps are equivalent to bipartite maps with only two-valent black vertices. Another interesting subcase is that of $p$-constellations with only $p$-valent black vertices and white vertices with valences that are multiples of $p[5]$. This corresponds to the most general situation where explicit compact formulas [15] are known for the numbers of maps with prescribed vertex valences. To complete the picture, note that 2 -constellations are equivalent to maps with even vertex valences.

On the other (dual) front, well-labeled trees appeared so far only in correspondence with quadrangulations [17] [12] and Eulerian (face-bicolored) triangulations [8] ${ }^{2}$. Here the labels on the trees correspond to a labeling of the vertices of the original map by their geodesic distance from a fixed origin vertex, which gives access to a host of results on the geometry of maps. This is to be contrasted with the blossom tree approach, where only two-point correlations (i.e. generating functions for maps with two points at a fixed geodesic distance) are within reach.

The aim of this paper is to extend this construction to the general case of Eulerian planar maps with prescribed face valences (dual to the bipartite planar maps with prescribed vertex valences considered in Ref. [4]) by introducing generalized labeled trees, which we call mobiles. In terms of generating functions, we reproduce and generalize the algebraic recursion relations of [6] and provide a new combinatorial interpretation for them.

\subsection{Plan of the paper}

The paper is organized as follows. For the sake of clarity, we begin in Sect. 2 by the simpler case of planar maps with even face valences. In Sect. 2.1, we associate to each such map with a distinguised vertex a mobile whose properties are further characterized. In Sect. 2.2, we display the inverse construction, proved in detail in Sect. 2.3. Finally, in Sect. 2.4 we derive equations for the corresponding generating functions, which determine a single function $R_{n}$ for maps with two points at a geodesic distance less than $n$.

In Sect. 3, we turn to the general case of Eulerian maps with prescribed face valences : we construct the corresponding mobiles in Sect. 3.1, and we exhibit the inverse construction in Sect. 3.2. Generating functions are studied in Sect. 3.3.

Sect. 4 is devoted to the illustration of the sub-cases of $p$-constellations and arbitrary maps, where mobiles may be simplified. A few concluding remarks on interesting applications are made in Sect. 5 .

2 Quadrangulations and Eulerian triangulations are respectively related to the original bijection of Cori and Vauquelin [13] and to that of Arquès [1], which both rely on an encoding of maps in terms of permutations. 


\section{The case of planar maps with prescribed even face degrees}

\subsection{From maps to mobiles}

We start from a planar map $\mathcal{M}$ whose faces all have even degrees. Equivalently, this amounts to requiring that the map be bipartite, namely that its vertices may be partitioned into two sets so that no two adjacent vertices belong to the same set. On this map, we distinguish a vertex as the origin and label all the vertices of the map by their geodesic distance to this origin, i.e. the length of any shortest path from the origin to that vertex. With this definition, the origin is the only vertex labeled 0 , its nearest neighbors are all labeled $1, \ldots$, and all the labels are non-negative integers. Moreover, any two adjacent vertices have labels differing by at most one, and because of the bipartite nature of the map, these labels cannot be equal.

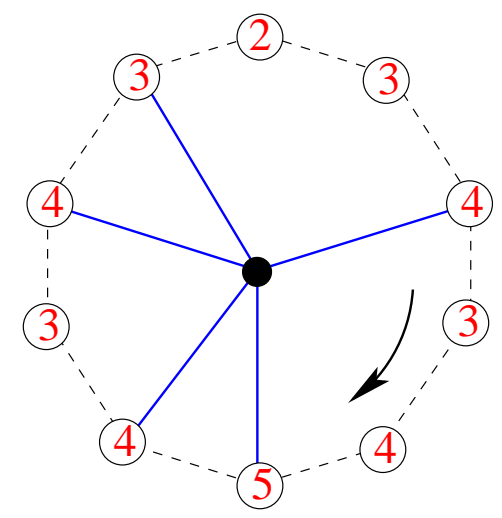

Fig. 1: A typical configuration of labels around a face of $\mathcal{M}$ (delimited by dashed edges), here of valence $2 k=10$. Adjacent labels differ by \pm 1 . We add a black unlabeled vertex at the center of the face and connect it (via solid edges) to the $k=5$ labeled vertices immediately followed clockwise by a smaller label.

Our construction takes place independently within each face of $\mathcal{M}$. Given a face of degree $2 k$, the adjacent vertex labels read in clockwise direction around the face form a cyclic sequence with increments of \pm 1 . Among these $2 k$ vertices, we select the $k$ ones immediately followed by vertices with smaller labels (see figure 1 for an example). We add a new (unlabeled) vertex at the center of the face and connect it within the face by $k$ new non-intersecting edges to the $k$ selected labeled vertices. After completing this construction within each face, we remove all the edges of the original map. We also erase the origin vertex as by construction it becomes isolated. We are left with a map $\mathcal{T}$ with two types of vertices: unlabeled ones in correspondence with the faces of $\mathcal{M}$, and labeled ones consisting of all the vertices of $\mathcal{M}$ except the origin. The edges of $\mathcal{T}$ connect only vertices of different types.

We now show that $\mathcal{T}$ is a plane tree. We first show that it contains no cycle and then that it has a single connected component. The first statement is proved by contradiction. Assume that $\mathcal{T}$ contains a cycle, namely a closed path separating the 


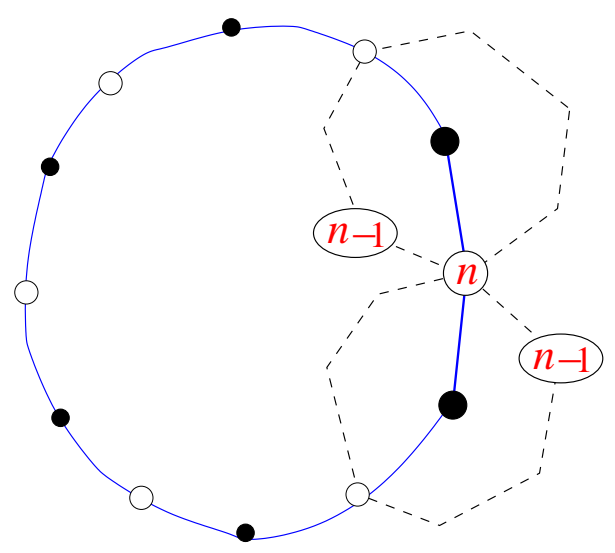

Fig. 2: Proof by contradiction that $\mathcal{T}$ has no cycle. Assuming the existence of such a cycle, we pick a labeled vertex with minimal label $n$ on the cycle. The neighborhoods of its two adjacent unlabeled vertices on the cycle show the existence of a vertex labeled $n-1$ on each side of the cycle. A path on $\mathcal{M}$ from the origin to one of these vertices must cross the cycle at a labeled vertex with label at most $n-1$, a contradiction.

plane into two regions. We call the interior of the cycle the region not containing the former origin. We pick a labeled vertex on the cycle whose label, say $n$, is minimal along the cycle. By examining the neighborhood of its two adjacent unlabeled vertices on the cycle (see figure 2), we conclude that there is a vertex labeled $n-1$ in the interior of the cycle. This is a contradiction: a geodesic path on $\mathcal{M}$ from the origin to this vertex must intersect the cycle at a labeled vertex with label $j \leq n-1<n$.

Having shown that $\mathcal{T}$ has no cycle, it is a forest made of $c$ trees. Let $V, F$ and $E$ denote respectively the numbers of vertices, faces and edges of $\mathcal{M}$, obeying the Euler relation $V-E+F=2$. The total number of vertices of $\mathcal{T}$ is $F+V-1$. The number of edges in $\mathcal{T}$ reads $\sum_{k} k F_{2 k}$ where $F_{2 k}$ is the number of $2 k$-valent faces of $\mathcal{M}$. This is nothing but $E$ as clearly $\sum_{k} 2 k F_{2 k}=2 E$. By noting that each of the $c$ trees has one more vertex than edge, we deduce that $c=(F+V-1)-E=1$. $\mathcal{T}$ is therefore a tree. An illustration of the complete construction of $\mathcal{T}$ starting from $\mathcal{M}$ is shown in figure 3 .

The labels of $\mathcal{T}$ have the following property:

(P) for each unlabeled vertex $v$, the labels $n$ and $m$ of two labeled vertices adjacent to $v$ and consecutive in clockwise direction satisfy $m \geq n-1$.

We define a mobile as a plane tree obeying the following rules:

(i) its vertices are of two types, unlabeled ones and labeled ones carrying integer labels,

(ii) each edge connects a labeled to an unlabeled vertex,

(iii) the labels obey the property $(\mathrm{P})$ above.

If the mobile moreover satisfies the additional rule:

(iv) all labels are strictly positive and there is at least one vertex with label 1 , then it will be said well-labeled. The reader may check that figure 3-(c) represents a well-labeled mobile.

The above construction maps each bipartite planar map with an origin into a well- 


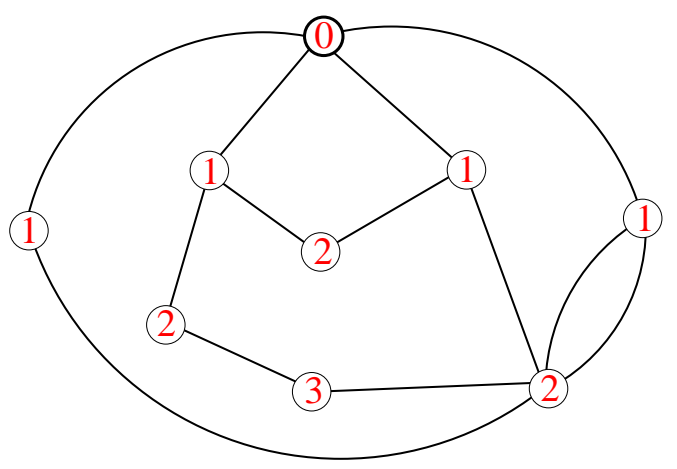

(a)

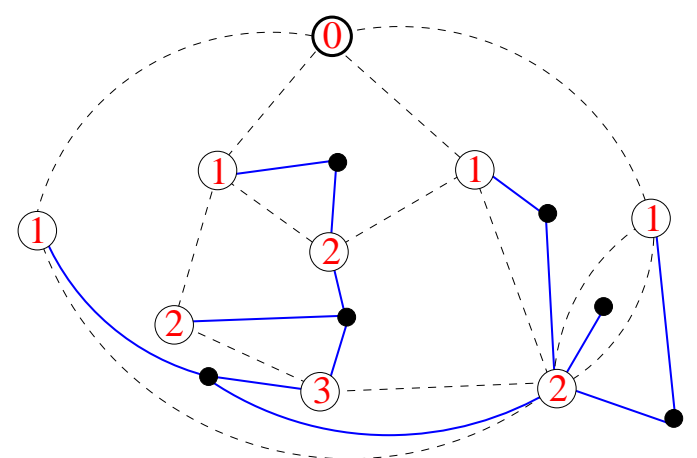

(b)

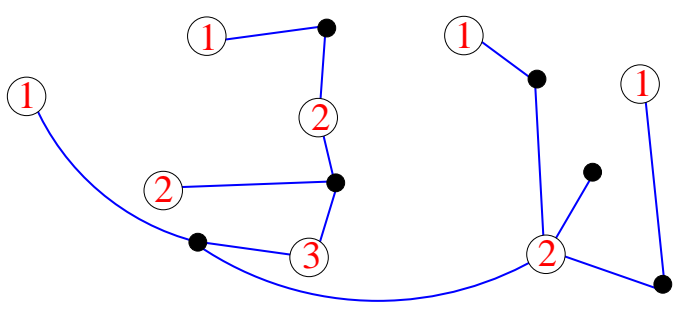

(c)

Fig. 3: A typical example of a planar map $\mathcal{M}$ (a) with one 2-valent, three 4 -valent and two 6-valent faces. Having selected an origin (labeled $0)$, all the vertices are naturally labeled by their geodesic distance to that origin. We perform (b) the construction of figure 1 in each face of $\mathcal{M}$ (with, as usual, the opposite conventions for the external face due to the representation in the plane). Erasing the original edges of $\mathcal{M}$ as well as its origin, we are left (c) with a tree $\mathcal{T}$.

labeled mobile. This mapping turns out to be a bijection whose inverse is exhibited in the next section.

\subsection{Converse construction}

We start from a well-labeled mobile $\mathcal{T}$. A corner of $\mathcal{T}$ is a sector with apex at a labeled vertex of $\mathcal{T}$ and delimited by two consecutive edges around this vertex. We label each corner by the label of its apex. To each corner $C$ with label $n \geq 2$, we associate its successor $s(C)$ defined as the first encountered corner with label $n-1$ when going clockwise around the tree (see figure 4-(b)). The existence of a successor is ensured by the property $(\mathrm{P})$. Indeed, at each step in the sequence of corners read clockwise around $\mathcal{T}$, the label may decrease by at most 1 ; hence between the corner $C$ and a corner with label 1 , all labels between 1 and $n$ must be present.

We construct the map $\mathcal{M}$ associated with $\mathcal{T}$ by first drawing an edge between each corner with label $n \geq 2$ and its successor within the external face of $\mathcal{T}$ and in such a 


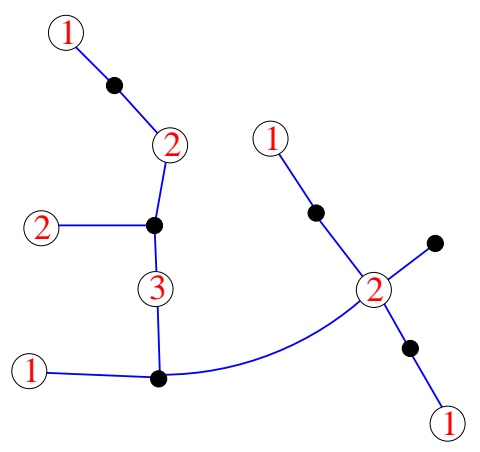

(a)

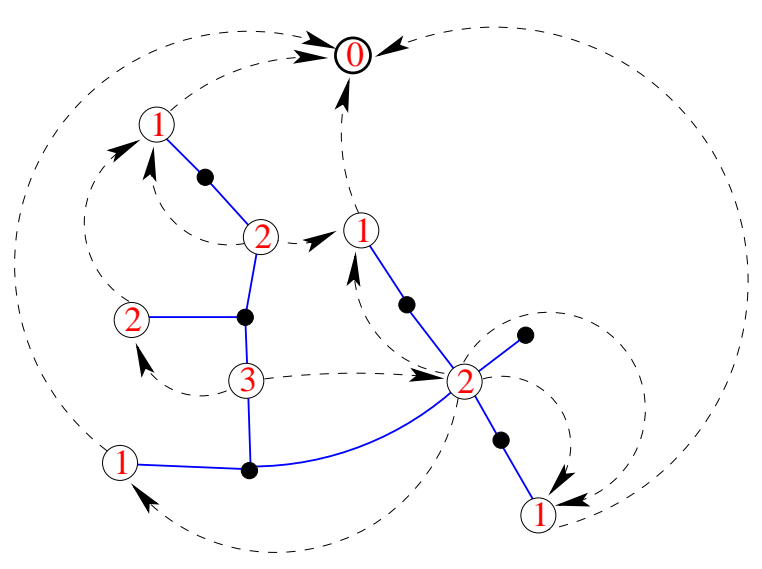

(b)

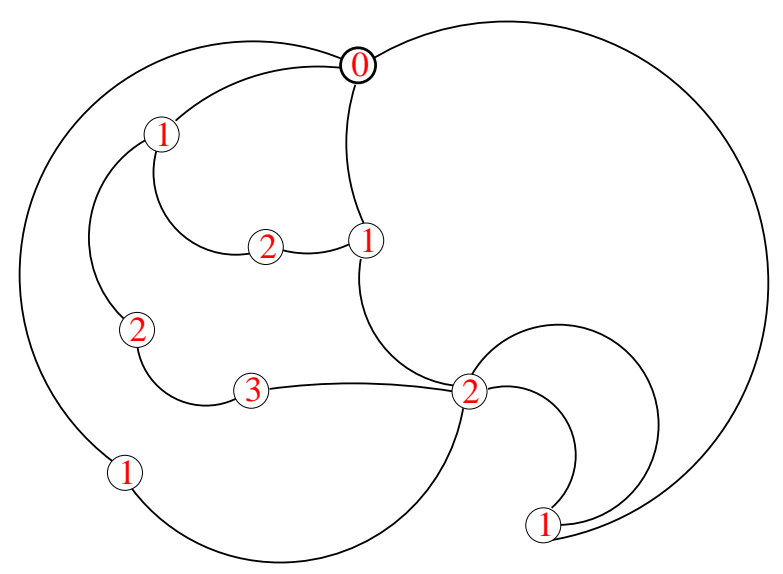

(c)

Fig. 4: A typical example of a well-labeled mobile $\mathcal{T}$ (a). For each unlabeled vertex of $\mathcal{T}$, the successive labels of its adjacent labeled vertices cannot decrease by more than 1 clockwise. We add an extra origin vertex labeled 0 and connect (b) each labeled corner to its successor (dashed arrows). Erasing all unlabeled vertices of $\mathcal{T}$ and their adjacent edges, we are left (c) with a bipartite planar map $\mathcal{M}$. Moreover, the labels simply encode the geodesic distance from the origin to the vertices.

way that no two edges intersect. This can be done due to the nested structure around $\mathcal{T}$ of corners and their successors, namely that if a corner $C^{\prime}$ lies strictly between a corner $C$ and its successor $s(C)$, then $s\left(C^{\prime}\right)$ lies between $C^{\prime}$ and $s(C)$ (with possibly $\left.s\left(C^{\prime}\right)=s(C)\right)$. Again this is a consequence of the property $(\mathrm{P})$. We next add an origin vertex labeled 0 in the external face and view the unique sector around this isolated point as the successor of all corners labeled 1, which we therefore also connect to the origin via non-crossing edges. This is possible because each corner has its successor before or at the first encountered corner labeled 1; hence all corners labeled 1 are incident to 
the external face. Finally we erase all unlabeled vertices and their adjacent edges. The result is a map $\mathcal{M}$ with an origin, which is connected because each vertex is connected to the origin via a chain of successors. It is moreover bipartite because the parity of labels alternate between adjacent vertices. We may forget about labels because these are nothing but the geodesic distances from the vertices of $\mathcal{M}$ to the origin. Indeed, since the labels on vertices adjacent in $\mathcal{M}$ differ by exactly 1 , the geodesic distance from a vertex to the origin is larger than or equal to its label $n$, and a chain of successors provides a geodesic of length $n$. Figure 4 displays an example of construction of the map $\mathcal{M}$ starting from a well-labeled mobile $\mathcal{T}$.

In the next section, we argue that the above construction is indeed the inverse of that presented in section 2.1.

\subsection{Proof of the bijection}

In order to prove that the two previous constructions are inverse of one another, we have to show successively the two following assertions:

(1) starting with a bipartite planar map $\mathcal{M}$ with an origin and constructing its associated mobile $\mathcal{T}$ as in section 2.1, the construction of section 2.2 carried out on this mobile $\mathcal{T}$ retrieves $\mathcal{M}$,

(2) starting with a well-labeled mobile $\mathcal{T}$ and constructing its associated map $\mathcal{M}$ as in section 2.2, the construction of section 2.1 carried out on this map $\mathcal{M}$ retrieves $\mathcal{T}$. To prove (1), we consider a bipartite map $\mathcal{M}$ with an origin and its associated mobile $\mathcal{T}$. We only have to prove that the construction of section 2.2 restores exactly the edges of $\mathcal{M}$, as the vertices of $\mathcal{M}$ are the labeled vertices in $\mathcal{T}$ or the origin. We consider an edge $e$ of $\mathcal{M}$ connecting two vertices labeled $n$ and $n-1$, say. In $\mathcal{T}$, the extremities of $e$ point to two corners of the union $\mathcal{T} \cup\{0\}$ of the tree $\mathcal{T}$ and the origin vertex $\{0\}$. We denote by $C$ the corner with label $n$ and by $C^{\prime}$ that with label $n-1$ : we have to show that $C^{\prime}$ is the successor of $C$. If $n=1$, this is automatic by construction. If $n \geq 2$, both $C$ and $C^{\prime}$ are true corners of $\mathcal{T}$. We consider the graph obtained by adding to $\mathcal{T}$ the edge $e$ : it has exactly 2 faces, one of which does not contain the origin. We follow the contour path $\mathcal{C}$ going counterclockwise around this face from one extremity of $e$ to the other. Let $m$ denote the smallest corner label on $\mathcal{C}$. Assume by contradiction that this label is attained at a corner which is not the last one on $\mathcal{C}$ : as shown in figure 5 , we deduce the existence of a corner on $\mathcal{C}$ with label $m-1<m$. Hence $m$ is only attained at the last corner on $\mathcal{C}$, which is therefore $C^{\prime}$ with label $m=n-1$, while the first corner is $C$ : as all labels in between are strictly larger than $n-1$, this proves that

$C^{\prime}$ is the successor of $C$, and thus $e$ is restored in the construction of section 2.2. A simple counting argument shows that the number of corners in $\mathcal{T}$ is exactly the number of edges in $\mathcal{M}$; hence the construction of section 2.2 does not create edges other than those of $\mathcal{M}$.

To prove (2), we consider a well-labeled mobile $\mathcal{T}$ and its associated map $\mathcal{M}$. We now characterize the faces of $\mathcal{M}$. We first show that each face of $\mathcal{M}$ contains exactly one unlabeled vertex of $\mathcal{T}$, and then that the rules of section 2.1 inside this face select precisely the edges of $\mathcal{T}$ incident to that vertex. Start with an unlabeled vertex of $\mathcal{T}$ 


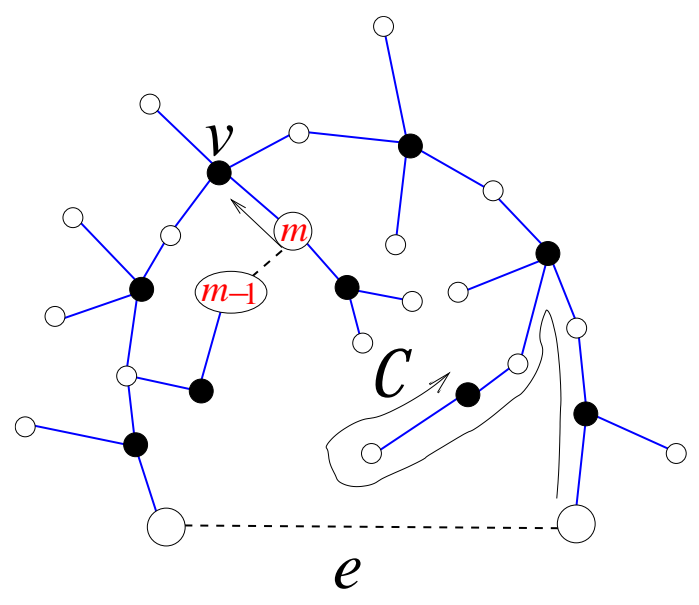

(0)

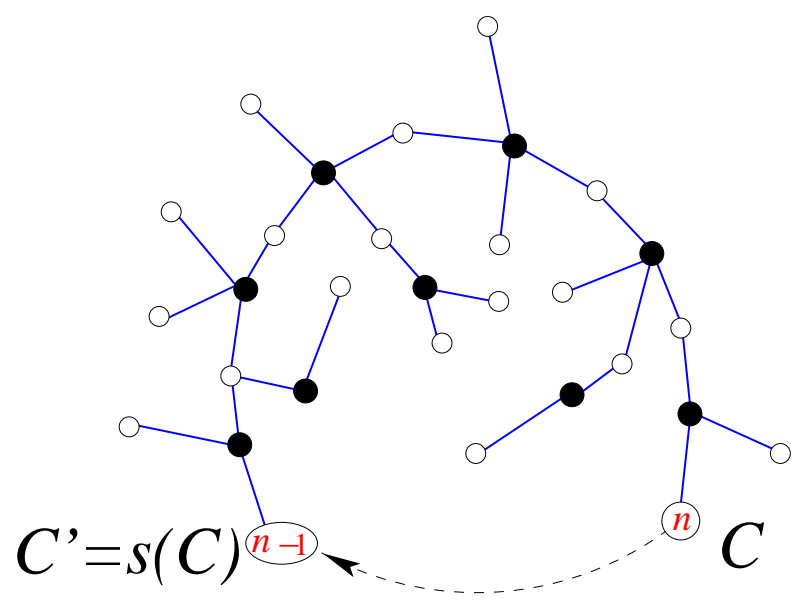

(b)

Fig. 5: Proof that any edge $e$ of $\mathcal{M}$ is restored in the construction of section 2.2. Apart from the trivial case where the origin is adjacent to $e$, the union of $e$ and $\mathcal{T}$ has two faces. We follow (a) counterclockwise the contour path $\mathcal{C}$ bordering the face not containing the origin and pick a corner with smallest label $m$ (represented here with an outgoing arrow). If this corner were not the last one on $\mathcal{C}$ (adjacent to $e$ ), it would be followed by an unlabeled vertex $v$ and by the selection rules of figure 1 around $v$, we would deduce the existence of another corner with label $m-1$ inside the face. As the face does not contain the origin, the corner $m-1$ is different from the origin hence it lies on the contour $\mathcal{C}$, a contradiction. This implies (b) that the end corner of $\mathcal{C}$ is the successor of its starting corner, hence that $e$ is restored.

and consider two clockwise consecutive adjacent corners as in figure 6-(a), with labels $n$ and $m$ (with $m \geq n-1$ by definition of a mobile). Then, the successor of the corner labeled $n$ belongs to the sequence of successors of the corner labeled $m$. This delimits a region of the plane which contains no other vertex or edge of $\mathcal{M}$. The union of these regions for all the corners adjacent to the unlabeled vertex at hand forms a face of $\mathcal{M}$ containing no other unlabeled vertex. All the faces of $\mathcal{M}$ are obtained this way, as shown by a counting argument: the numbers of edges in $\mathcal{T}$ and $\mathcal{M}$ are equal (and both equal to the number of labeled corners in $\mathcal{T}$ ), and there is one more vertex in $\mathcal{M}$ as labeled vertices in $\mathcal{T}$. Using Euler's relation both for $\mathcal{M}$ and $\mathcal{T}$ shows that there are as many faces in $\mathcal{M}$ than unlabeled vertices in $\mathcal{T}$. Finally, as apparent from figure $6-(\mathrm{b})$, the selection rules of section 2.1 select precisely the vertices originally connected in $\mathcal{T}$ to the same unlabeled vertex. 


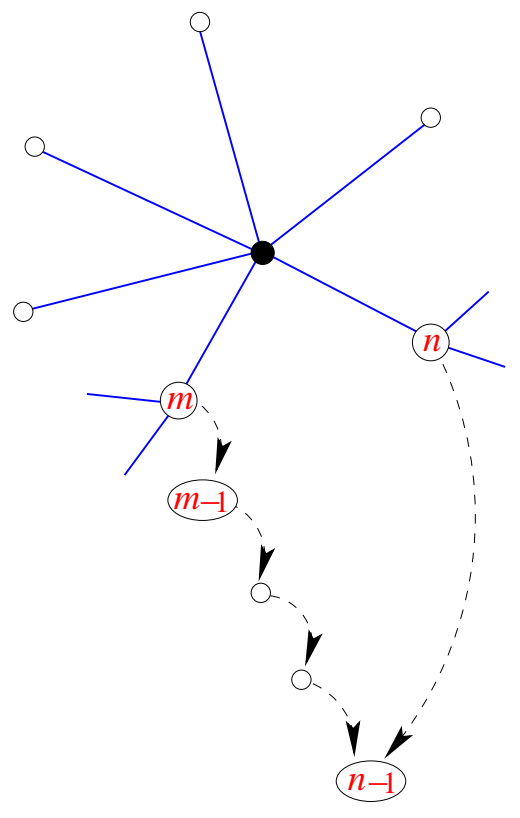

(a)

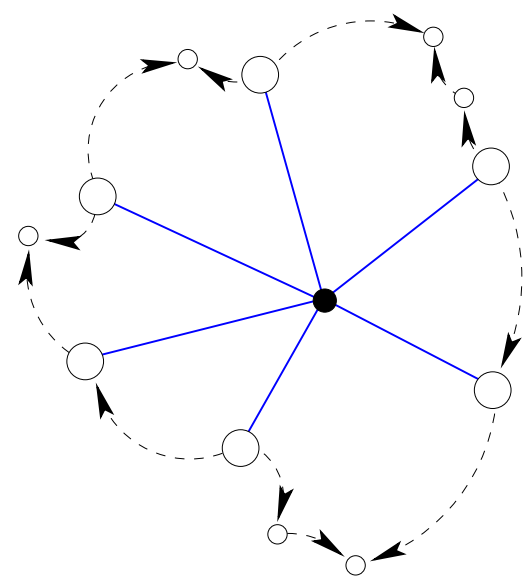

(b)

Fig. 6: The generic configuration (a) of successors of two consecutive vertices adjacent to a given unlabeled vertex of $\mathcal{T}$. Each face of $\mathcal{M}$ is obtained (b) as the union around an unlabeled vertex of all such configurations. As the labels decrease by one along the arrows, we immediately see that the labels of $\mathcal{T}$ are re-selected by the construction of section 2.1 acting on $\mathcal{M}$.

\subsection{Generating functions}

The constructions of Sects. 2.1 and 2.2 establish a bijection between, on the one hand, bipartite planar maps with an origin vertex, and, on the other hand, well-labeled mobiles. Enumeration is simpler for rooted mobiles, which enjoy recursive properties. More precisely, a rooted mobile has a distinguished corner, which in terms of maps corresponds to distinguishing an edge. We may moreover attach weights $g_{2 k}$ per $k$ valent unlabeled vertex of the mobile, which amounts to a weight $g_{2 k}$ per $2 k$-valent face of the planar maps.

Let $R_{n} \equiv R_{n}\left(\left\{g_{2 k}\right\}\right)$ denote the generating function for rooted mobiles with root corner labeled $n$ (see figure 7 ). Splitting a mobile at its root vertex leads to an arbitrary number of sub-mobiles, which implies the relation

$$
R_{n}=\frac{1}{1-L_{n}}
$$

where $L_{n}$ denotes the generating function for mobiles rooted at a univalent vertex labeled $n$. These new objects may be decomposed according to the sequence of labels around the unlabeled vertex adjacent to the root. By definition of mobiles, such a sequence of 

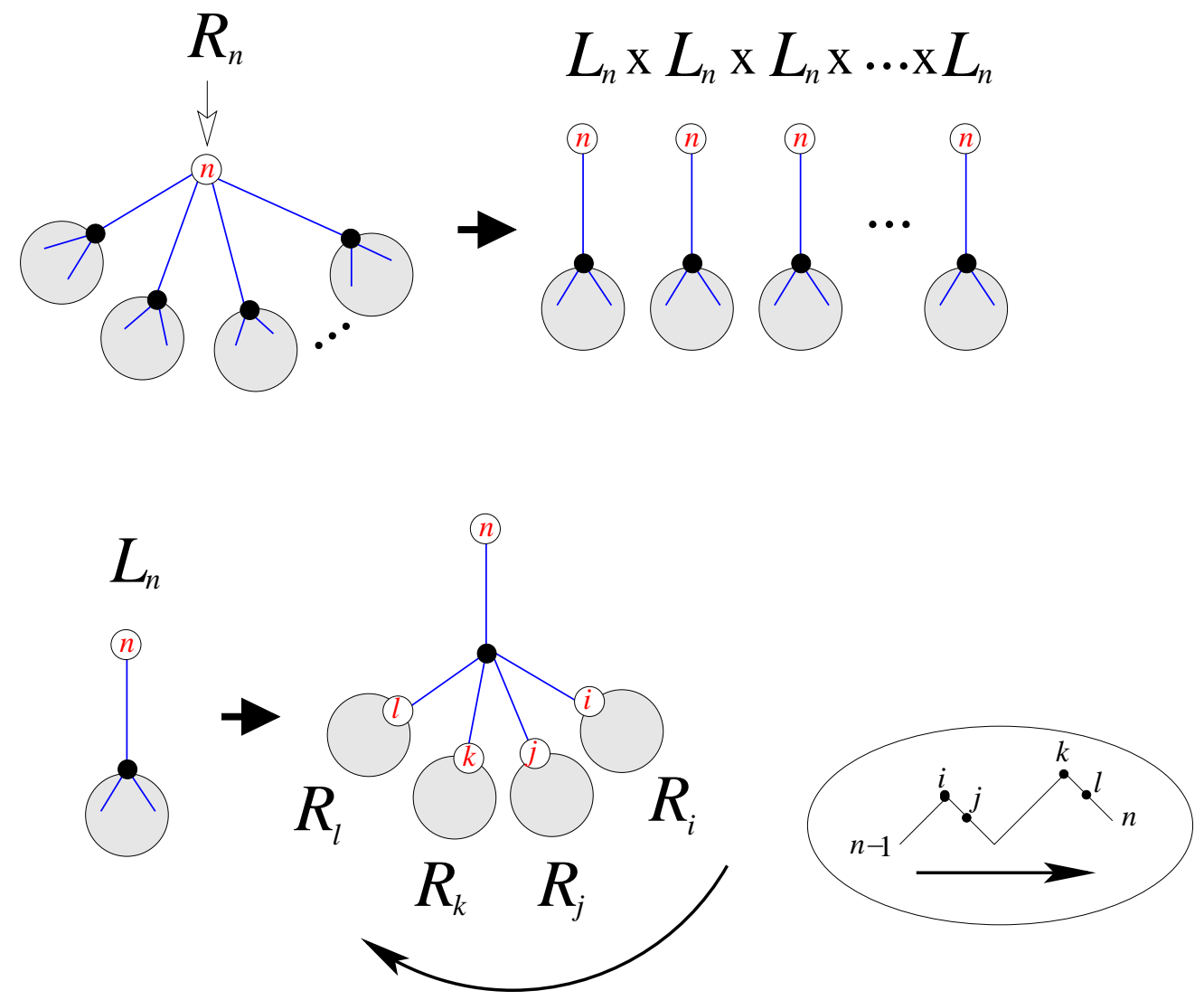

Fig. 7: Top: the decomposition of a rooted mobile with root label $n$ (generating function $R_{n}$ ) into a number of mobiles with univalent root vertex with the same label (generating function $L_{n}$ ) leads to eq. (2.1). Bottom: A mobile with univalent root vertex with label $n$ is in turn decomposed according to the clockwise neighborhood of the adjacent unlabeled vertex, in correspondence with walks (see oval). Each descending step $i \rightarrow i-1$ on the walk gives rise to a factor $R_{i}$ for the associated rooted sub-mobile, leading to eq. (2.2).

length $k$ is in correspondence with a walk of length $2 k-1$ on the integer line with steps of \pm 1 , starting at position $n-1$ and ending at position $n$. To each descending step $m \rightarrow m-1$ is associated a mobile rooted at a corner $m$ (see figure 7 ). This implies that

$$
L_{n}=\sum_{k=1}^{\infty} g_{2 k}\left\langle n\left|Q^{2 k-1}\right| n-1\right\rangle \text {, }
$$

where $Q$ is the operator acting on a formal orthonormal basis $|i\rangle, i \in \mathbb{Z}$ (with $\langle j \mid i\rangle=\delta_{i j}$ ) as

$$
Q|i\rangle=|i+1\rangle+R_{i}|i-1\rangle \text {. }
$$

The operator $Q$ may be understood as a transfer matrix acting clockwise around unlabeled vertices. 
For instance, the first few valences contribute as

$$
\begin{aligned}
\langle n|Q| n-1\rangle & =1 \\
\left\langle n\left|Q^{3}\right| n-1\right\rangle & =R_{n}+R_{n+1}+R_{n-1} \\
\left\langle n\left|Q^{5}\right| n-1\right\rangle & =R_{n+1} R_{n+2}+R_{n+1} R_{n-1}+R_{n-1} R_{n-2} \\
& +R_{n+1}^{2}+R_{n}^{2}+R_{n-1}^{2}+2 R_{n}\left(R_{n+1}+R_{n-1}\right) .
\end{aligned}
$$

In the case of well-labeled mobiles, the positivity of labels is enforced by imposing $R_{-i}=$ 0 for $i=0,1,2, \cdots$, and by considering Eqs. (2.1)-(2.3) for $n \geq 1$ only. With these appropriate boundary conditions, these equations form a closed set determining formally the $R_{n}$ 's and $L_{n}$ 's as power series in the $g_{2 k}$ 's. The presence of at least one label 1 is guaranteed by considering the quantity $R_{n}-R_{n-1}$, which is therefore identified with the generating function for well-labeled mobiles with a distinguished corner with label $n$. Thanks to our bijection, it is also the generating function for bipartite planar maps with an origin and a distinguished edge of type $n-1 \rightarrow n$. As $n \rightarrow \infty$, the limit $R$ of $R_{n}$ corresponds to the generating function for bipartite planar maps with an origin and a distinguished edge, and satisfies

$$
R=\frac{1}{1-\sum_{k=1}^{\infty}\left(\begin{array}{c}
2 k-1 \\
k
\end{array}\right) g_{2 k} R^{k-1}} .
$$

Alternatively, $R$ is also the generating function for (non-necessarily well-labeled) mobiles rooted at a corner labeled, say, 0, which has a clear bijective justification. Similarly, using $\sum_{k \geq 1}(1 / k) L_{n}^{k}=\log R_{n}$, we find that the function $\log \left(R_{n} / R_{n-1}\right)$ for $n \geq 2$ (resp. $\log R_{1}$ for $n=1$ ) generates bipartite planar maps with an origin and a distinguished vertex at geodesic distance $n$, weighted by their inverse symmetry factor.

As a final remark, note that equivalent equations have been derived by the use of an alternative bijection using so-called blossom trees in Ref. [6], where explicit solutions have been found, due to a remarkable integrability property.

\section{General case: Eulerian maps with prescribed face degree distribution}

In this section, we extend the construction of section 2 to a more general class of maps, namely Eulerian, i.e. face-bicolored maps with fixed numbers of faces with prescribed color and degree. This generalizes the previous case which may indeed be recovered by imposing that all the faces of a given color, black say, have degree two, and that all the faces of the other color, white say, have an even degree, and by contracting all the black faces into single edges.

\subsection{From Eulerian maps to generalized mobiles}

In this section, we show how to associate to an Eulerian map $\mathcal{M}$ a labeled tree $\mathcal{T}$ that we call a generalized mobile, characterized by specific label constraints. 
Geodesic distance in an Eulerian map $\mathcal{M}$ :

We start with an Eulerian map $\mathcal{M}$ and as before select an origin vertex. Every edge receives a natural orientation by requiring that the incident black face sits on its right. We now label each vertex of $\mathcal{M}$ by its oriented geodesic distance from the origin, namely the length of any shortest path respecting the edge orientations from the origin to this vertex. Such an oriented path always exists because, starting from an unoriented path, we may bypass any edge that points in the wrong direction by circumventing an incident face. The origin is the only vertex labeled 0 and all the labels are non-negative. Moreover, by the geodesic requirement, the labels $m$ and $n$ on, respectively, the starting-point and the end-point of any oriented edge must satisfy $n \leq m+1$.

Construction rules for $\mathcal{T}$ :
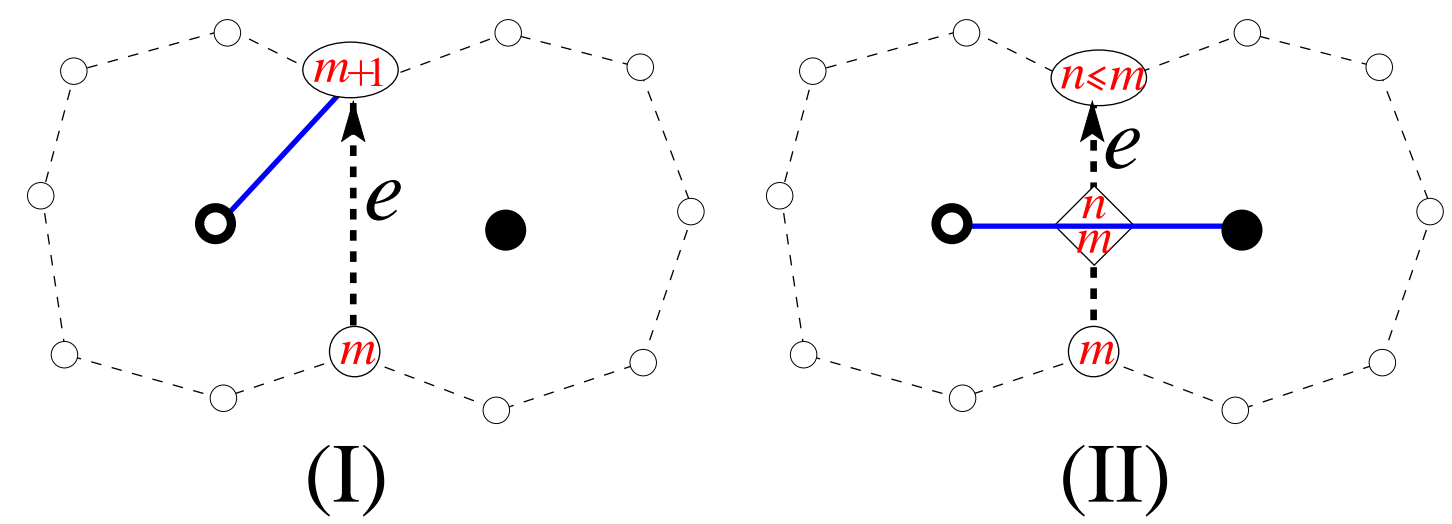

Fig. 8: Rules to construct a tree $\mathcal{T}$ out of an Eulerian map $\mathcal{M}$. We first add an unlabeled vertex at the center of each face of the map, with the same color (white or black), represented by thick (empty or filled) circles. Each oriented edge $e$ of $\mathcal{M}$ must connect vertices with labels of the form (I) $m \rightarrow m+1$ or (II) $m \rightarrow n$, with $n \leq m$. Accordingly, we draw an edge of $\mathcal{T}$ respectively connecting (I) the white unlabeled vertex on the left of $e$ to the vertex labeled $m+1$ and (II) the white and black unlabeled vertices on both sides of $e$. In the latter case we decorate the edge with labeled flags carrying the labels of the closest vertices.

The construction begins by adding a new unlabeled vertex at the center of each face of $\mathcal{M}$. Each unlabeled vertex receives the color of the face it lies in. We then consider independently each oriented edge $e$ of $\mathcal{M}$ and as above denote by $m$ and $n$ the labels of its starting- and end-points. We apply one of the following constructions:

(I) if $n=m+1$, we draw a new edge from the end-point of $e$ to the white unlabeled vertex in the white face at the left of $e$ (see figure 8-(I)).

(II) if $n \leq m$, we drawn a new edge cutting $e$ and linking the unlabeled (black and white) vertices in both faces adjacent to $e$. On each side of this new edge, we add a flag carrying the label $m$ or $n$ of the extremity of $e$ lying on this side (see figure 8-(II)). 
After completing this construction for each edge of $\mathcal{M}$, we remove all these original edges. We also erase the origin vertex because by construction it becomes isolated. We are left with a graph $\mathcal{T}$ made of three types of vertices:

- labeled vertices consisting of all the vertices of $\mathcal{M}$ except the origin,

- unlabeled black vertices in correspondence with the black faces of $\mathcal{M}$,

- unlabeled white vertices in correspondence with the white faces of $\mathcal{M}$. The edges of $\mathcal{T}$ are of two types:

- flagged edges with two flags, one on each side, connecting black to white unlabeled vertices

- unflagged edges connecting white unlabeled vertices to labeled ones.

Figure 9 shows an example of our construction on a sample Eulerian map.

Proof that $\mathcal{T}$ is a tree:

As before, $\mathcal{T}$ is a plane tree: it has no cycles and a single connected component. For the first statement, assume by contradiction the existence of a cycle, delimiting an interior domain not containing the former origin. We now consider the smallest label $n$ among

- the labels of the labeled vertices belonging to the cycle,

- the labels on the flags along the cycle and lying in the interior.

If $n$ is attained for a labeled vertex on the cycle, we deduce from the construction (I) that there is a vertex labeled $n-1$ in the interior. Consider an oriented geodesic path on $\mathcal{M}$ to this latter vertex: it necessarily intersects the cycle at a labeled vertex since all its edges are of type (I) above hence they never cut an edge of $\mathcal{T}$. The label at the intersection is strictly smaller than $n$. Otherwise, if $n$ is not attained for a labeled vertex, but only by flags, we deduce from the construction (II) the existence of vertex labeled $n$ in the interior, hence by considering again a geodesic path to this latter vertex, the existence of a vertex with label $\leq n$ on the cycle. In both cases, we have a contradiction.

The connectedness is proved as before by a counting argument: since $\mathcal{T}$ contains no cycle, its number $c$ of connected components is the difference between its number of vertices and its number of edges. Denoting by $V, E$, and $F$ the numbers of vertices, edges and faces of the original Eulerian map $\mathcal{M}$, the number of edges of $\mathcal{T}$ is obviously $E$ by construction, while the number of its vertices is $V+F-1$; hence $c=1$ by the Euler relation for $\mathcal{M}$. This concludes the proof that $\mathcal{T}$ is a plane tree.

Label characterization of $\mathcal{T}$ :

We now investigate the properties of labels in $\mathcal{T}$ inherited from those of $\mathcal{M}$. Each black unlabeled vertex is incident to flagged edges only. The flag labels have the following property:

(P•) for each black unlabeled vertex of $\mathcal{T}$, the sequence of flag labels of its incident edges, when read clockwise, is non-increasing at each edge crossing and non-decreasing between two consecutive edges (see figure 10).

Each white unlabeled vertex is incident to both flagged edges and unflagged edges leading to labeled vertices. The labels have the following property:

$\left(\mathrm{P}_{\circ}\right)$ for each white unlabeled vertex, the sequence of labels on incident flagged edges 

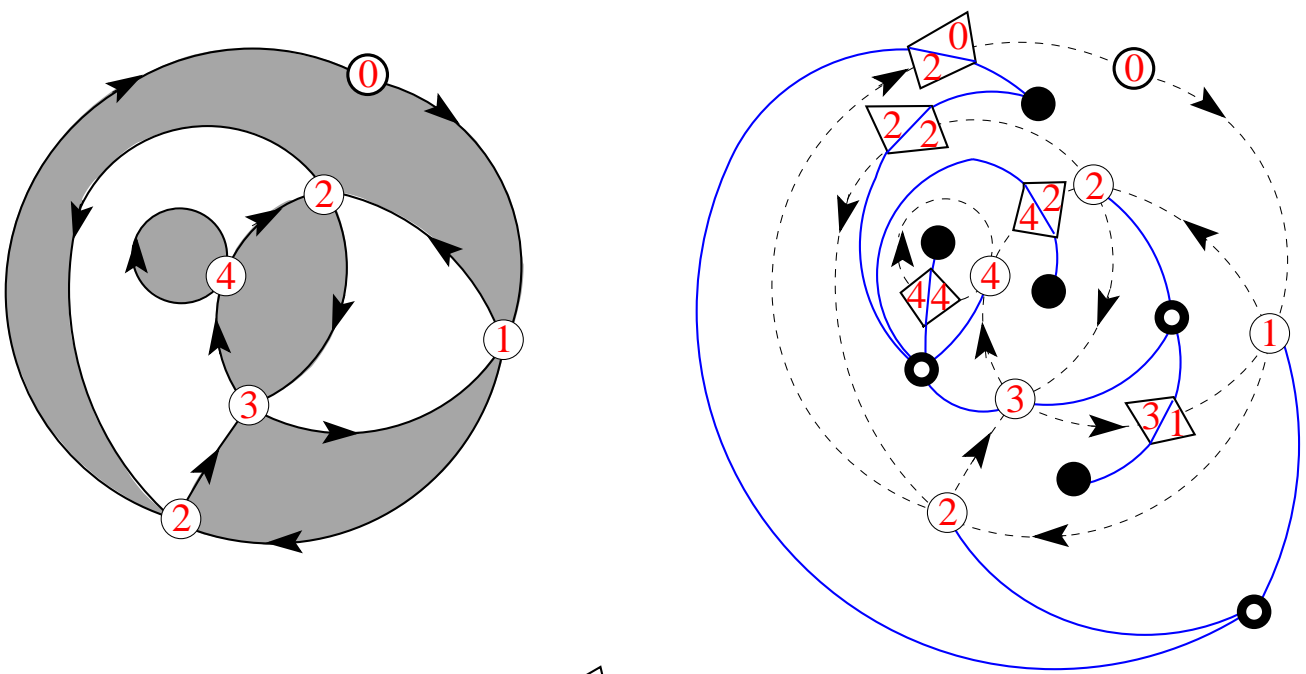

(a)

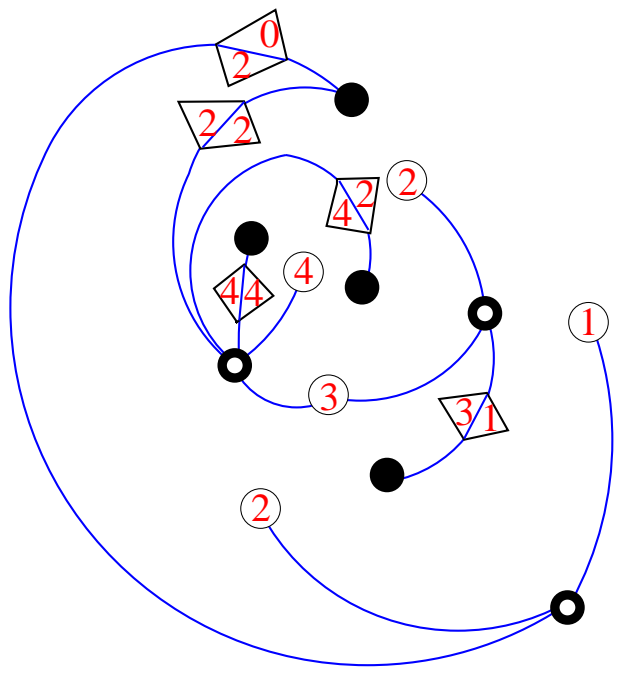

(b)

(c)

Fig. 9: The construction of a tree $\mathcal{T}$ out of a sample Eulerian map $\mathcal{M}$ with an origin. (a) Each vertex of $\mathcal{M}$ is labeled by its oriented geodesic distance from the origin 0 . (b) We perform the construction of figure 8 (I)-(II) for each oriented edge of the map. (c) Erasing the origin and the original edges of $\mathcal{M}$ produces a tree $\mathcal{T}$ carrying labels on some vertices and edges.

and on adjacent labeled vertices, when read clockwise, is non-decreasing at each edge crossing, decreasing by 1 after each labeled vertex and stationary between a flagged edge and the next label (see figure 11).

$P_{\bullet}$ and $P_{\circ}$ simply rephrase the properties of labels around faces of $\mathcal{M}$, namely that the clockwise sequence of labels around a black (resp. white) face are in one-to-one correspondence with closed walks with ascending steps of arbitrary (possibly zero) integer height, and descending steps of -1 . The walk must be read from left to right (resp. 

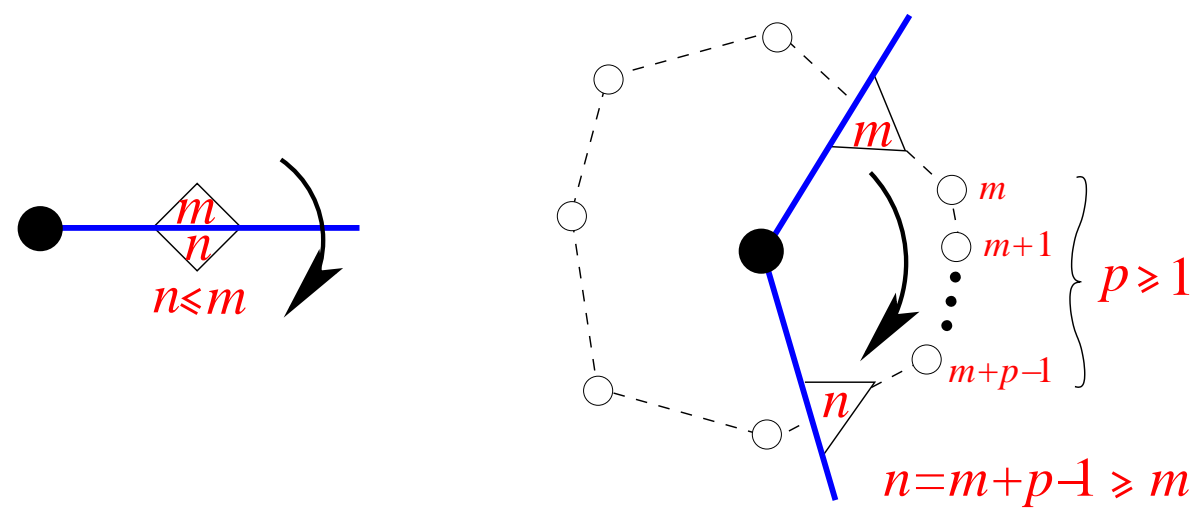

Fig. 10: Constraints $P_{\bullet}$ on labels around a black unlabeled vertex. Clockwise around the vertex two successive (flag) labels $m$ and $n$ must either satisfy $n \leq m$ if the flags share the same edge, or $n \geq m$ otherwise. In the latter case, $p=n-m+1$ may be interpreted as the number of labeled vertices incident in $\mathcal{M}$ to the corresponding black face, and in between the two flags.

right to left) in the case of a white (resp. black) face, as indicated in figure 12 . Note also that the valence of the faces of $\mathcal{M}$ is nothing but the length (number of steps) of the corresponding walks. As shown in figure 12, the coding of a walk in terms of tree labels is slightly different around black and white vertices.

We define a generalized mobile to be a plane tree such that:

(i) its vertices are of three types: black unlabeled vertices, white unlabeled vertices and labeled vertices carrying integer labels,

(ii) its edges are of two types: flagged edges with one flag on each side carrying an integer label, which connect unlabeled vertices of different colors, and unflagged edges, each connecting a labeled vertex to a white unlabeled one,

(iii) the labels obey the properties $\left(\mathrm{P}_{\bullet}\right)$ and $\left(\mathrm{P}_{\circ}\right)$ above.

If the mobile moreover satisfies the additional rule:

(iv) all vertex labels are strictly positive, all flag labels are non-negative, and there is at least a flag labeled 0 ,

then it will be called well-labeled.

The above construction provides a mapping from Eulerian planar maps to welllabeled generalized mobiles. This mapping is a bijection whose inverse is constructed in the next section.

\subsection{Inverse construction}

We start from a well-labeled generalized mobile $\mathcal{T}$. As before, we define a corner of $\mathcal{T}$ as a sector with apex at a labeled vertex and delimited by two consecutive (unflagged) incident edges. Each corner receives the label of its apex. We now define successors for both corners and flags:

- for each corner $C$ with label $n \geq 2$, its successor $s(C)$ is the first encountered corner with label $n-1$ when going clockwise around the tree, 

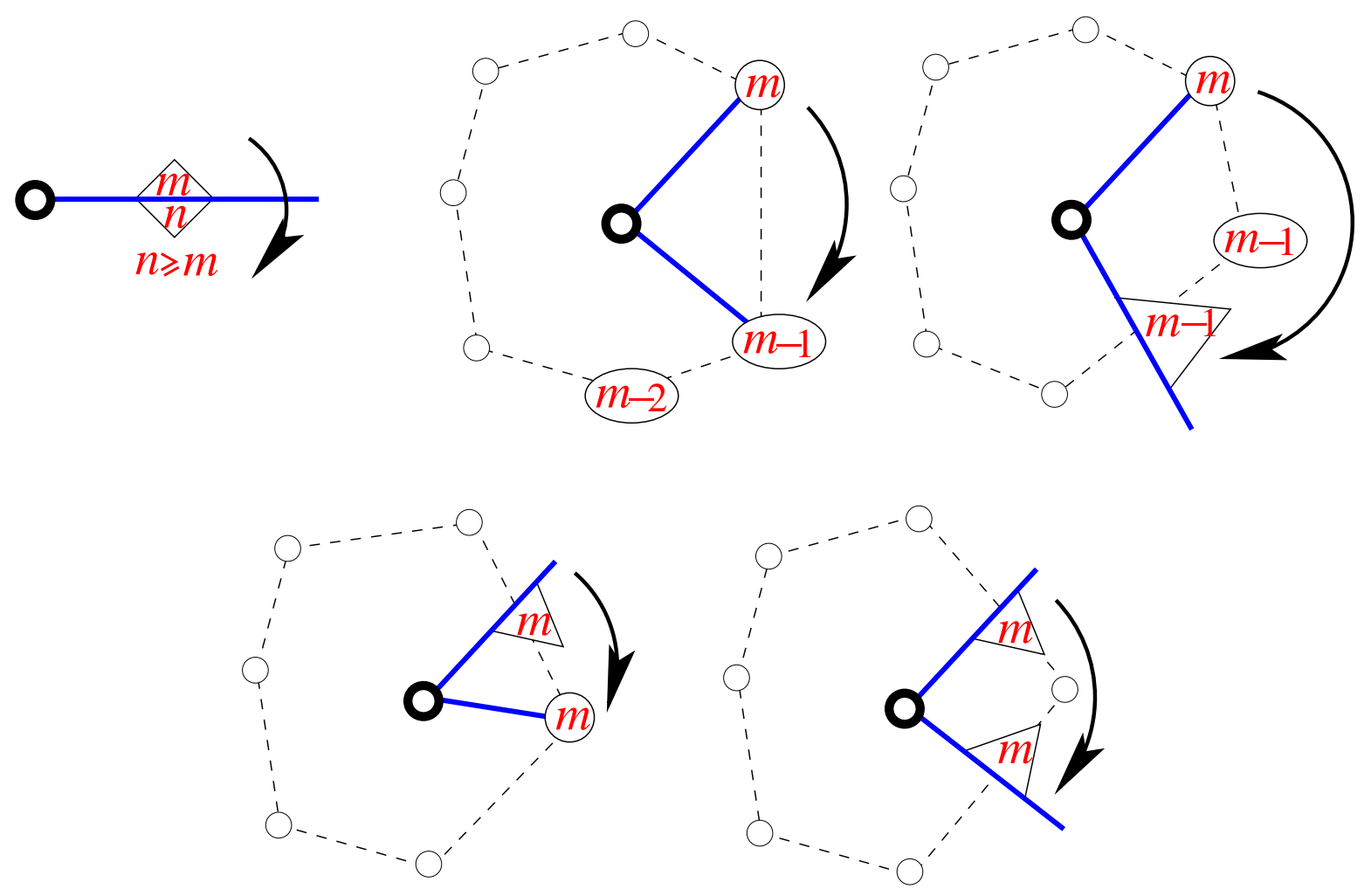

Fig. 11: Constraints $P_{\circ}$ on labels around a white unlabeled vertex read clockwise. Two successive flag labels $m$ and $n$ sharing the same edge must satisfy $n \geq m$. For consecutive edges, a vertex labeled $m$ is followed by a label $m-1$ (either on a vertex or a flag), while a flag labeled $m$ is followed by a label $m$ (either on a vertex or a flag).

- for each flag $F$ with label $n \geq 1$, its successor $s(F)$ is the first encountered corner with label $n$ when going clockwise around the tree.

The existence of successors is a consequence of the rules (iii) and (iv) defining welllabeled generalized mobiles. Indeed, the sequence of labels read clockwise around $\mathcal{T}$ is non-decreasing after a flag, and decreases by one after a corner. Hence between a corner with label $n \geq 2$ and a flag labeled 0 , there is at least one label $n-1$ after which the sequence decreases, thus corresponding to a corner. Similarly between a flag with label $n \geq 1$ and a flag labeled 0 , there is at least one label $n$ after which the sequence decreases, again corresponding to a corner.

We construct the map $\mathcal{M}$ associated to $\mathcal{T}$ by connecting each corner with label $\geq 2$ and each flag with label $\geq 1$ to its successor, which can be done in such a way that these newly created edges do not intersect. This is possible because of the nested structure of successors, a consequence of the above property of the label sequence around $\mathcal{T}$. At this point, all the corners labeled 1 and flags labeled 0 are in the same external face and we connect them all to a new origin vertex labeled 0 inside this face. Finally we erase all the edges of $\mathcal{T}$, whether flagged or unflagged, as well as all the unlabeled vertices, whether black or white. The result is a map $\mathcal{M}$ with an origin which is connected as 

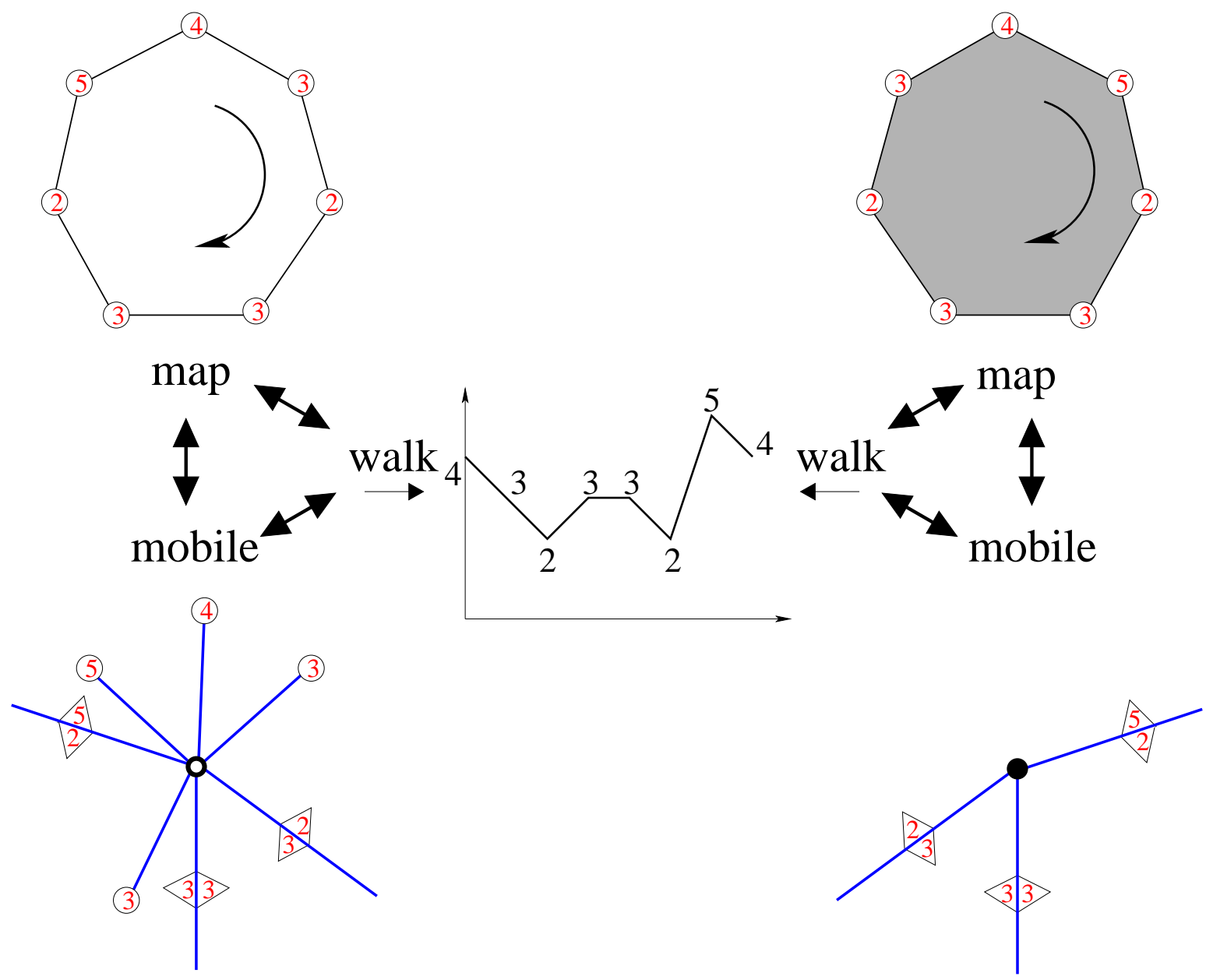

Fig. 12: The coding of the sequence of labels around white (left) and black (right) faces of $\mathcal{M}$ (top) and unlabeled vertices of $\mathcal{T}$ (bottom) via a closed walk (center) with increments $\geq-1$. The successive labels around faces of $\mathcal{M}$ when read clockwise match the successive heights of the walk when read from left to right (white faces) or right to left (black faces). The vertex labels around an unlabeled white vertex of $\mathcal{T}$ match the heights preceding steps of -1 on the walk, while flag labels match all other steps, of non-negative integer height. The flag labels around an unlabeled black vertex of $\mathcal{T}$ match the heights of all steps of non-positive integer height.

each vertex is connected to the origin via a chain of successors.

With the help of figure 13, the reader can easily check that $\mathcal{M}$ is indeed an Eulerian map, and that its vertices are labeled by their oriented geodesic distance from the origin. Moreover, the above construction and that of Sect. 3.1 are the inverse of one another. This can be proved by following the same line of arguments of in Sect. 2.3, suitably generalized so as to include the flagged edges. We omit the details here. 


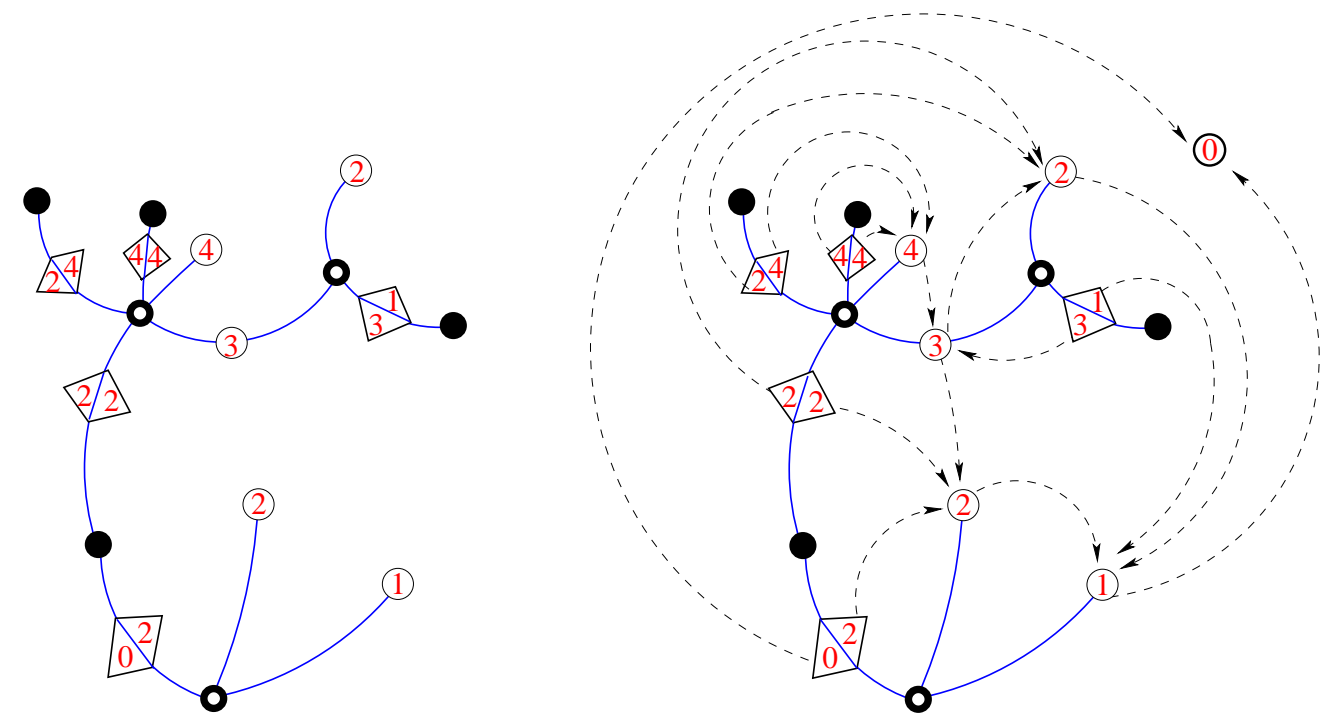

(a)

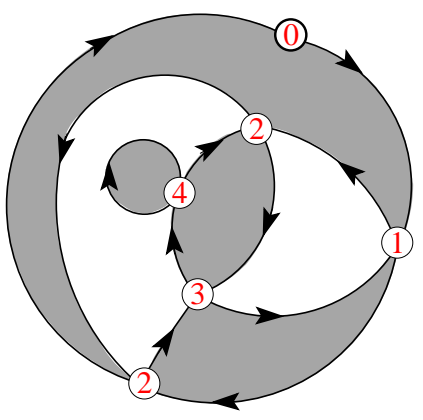

(b)

(c)

Fig. 13: The construction of an Eulerian map $\mathcal{M}$ with an origin out of a sample well-labeled generalized mobile $\mathcal{T}$. Starting from the mobile (a) we connect each labeled corner and flag to its successor via an oriented edge (dashed arrow). This includes adding a vertex labeled 0, successor of all corners labeled 1 and flags labeled 0. Erasing all unlabeled vertices and all edges of the mobile produces an Eulerian planar map with an origin (c). Its vertex labels moreover encode their geodesic distance from the origin.

\subsection{Generating functions}

As in section 2.4, we may derive recursion relations for the generating functions for mobiles, also interpreted as generating functions for Eulerian maps. Again we attach weights $g_{k}$ (resp. $\tilde{g}_{k}$ ) to white (resp. black) $k$-valent faces of the Eulerian map.

More precisely, let $R_{n}$ denote the generating function for rooted mobiles, i.e. with a distinguished corner labeled $n$. As in section 2.4, by splitting such a mobile at its root 


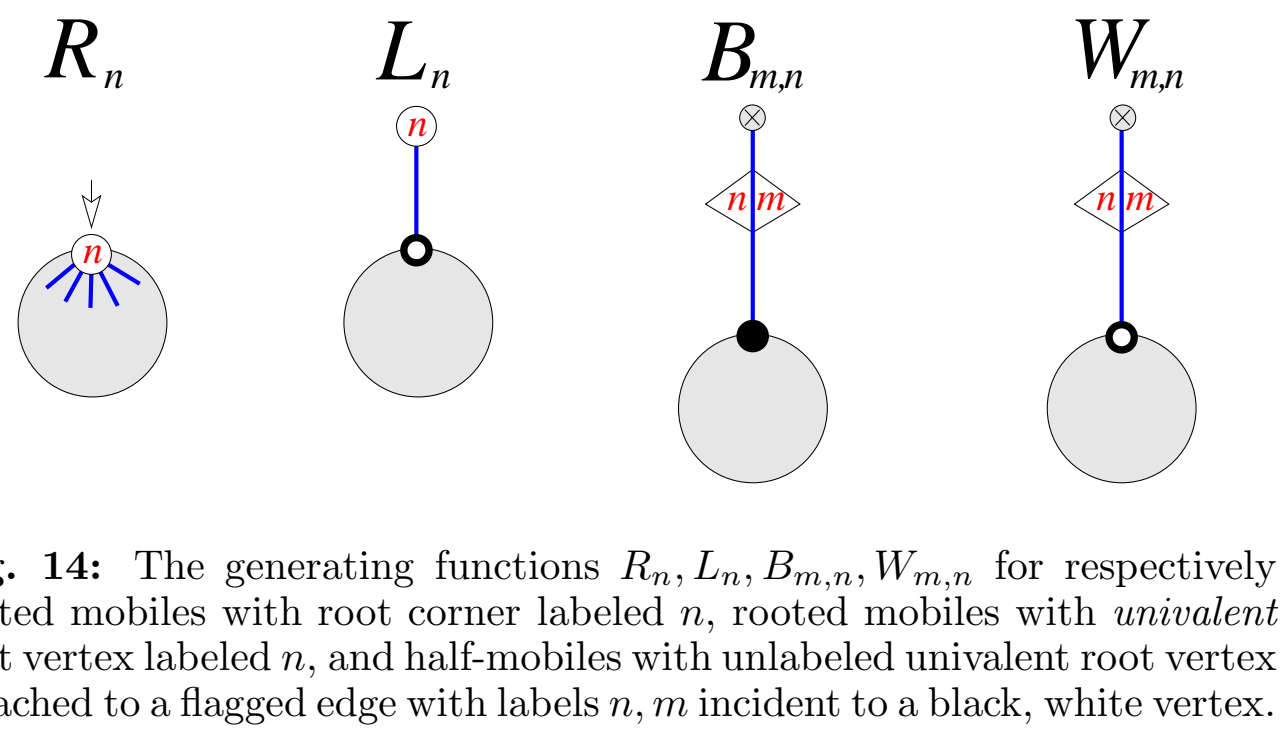

vertex, we obtain the equation

$$
R_{n}=\frac{1}{1-L_{n}},
$$

where $L_{n}$ denotes the generating function for mobiles rooted at a univalent vertex labeled $n$. To proceed further in the decomposition of these objects, we are led to introduce new classes of objects, namely half-mobiles, obtained by cutting a mobile across a flagged edge. These are represented in figure 14 as trees starting with a univalent unlabeled root vertex incident to a flagged edge. The rules $P_{\circ}$ and $P_{\bullet}$ are obeyed around each unlabeled vertex except the root. We denote by $W_{m, n}$ (resp. $B_{m, n}$ ) the generating function for half-mobiles whose root vertex is adjacent to a white (resp. black) unlabeled vertex via a flagged edge with flag $m$ on the right and $n$ on the left when going towards the root (see figure 14). By the rules $P_{\circ}$ and $P_{\bullet}, W_{m, n}=0$ for $m<n$, while $B_{m, n}=0$ for $m>n$.

By decomposing a mobile rooted at a univalent vertex labeled $n$ around its adjacent unlabeled white vertex, we obtain the recursive relation

$$
L_{n}=\sum_{k} g_{k}\left\langle n\left|Q^{k-1}\right| n-1\right\rangle,
$$

where $Q$ is the operator acting on a formal orthonormal basis as

$$
Q|i\rangle=\sum_{j \geq i} B_{i, j}|j\rangle+R_{i}|i-1\rangle
$$

which generates the sum over all walks from height $n-1$ to height $n$ with steps $\geq-1$ and with weights

- $R_{i}$ per step $i \rightarrow i-1$

- $B_{i, j}$ per step $i \rightarrow j$ with $j \geq i$

- $g_{k}$ per walk of length $k-1$ (corresponding to a $k$-valent white face). 
This follows from the correspondence displayed in figure 12 between white vertex neighborhoods in mobiles, and walks : the operator $Q$ may be understood as a transfer matrix acting clockwise around white vertices and therefore corresponds to reading walks from left to right in figure 12 .

Similar recursive relations for $W_{m, n}$ with $m \geq n$ follow from the same considerations, leading to

$$
W_{m, n}=\sum_{k} g_{k}\left\langle n\left|Q^{k-1}\right| m\right\rangle .
$$

Finally, repeating the exercise around black vertices leads to the following recursive relation valid for $m \leq n$

$$
B_{m, n}=\sum_{k} \tilde{g}_{k}\left\langle n\left|\tilde{Q}^{k-1}\right| m\right\rangle
$$

where $\tilde{Q}$ acts as

$$
\tilde{Q}|i\rangle=|i+1\rangle+\sum_{j \leq i} W_{i, j}|j\rangle,
$$

and may be interpreted as a transfer matrix generating black vertex neighborhoods in the clockwise direction, or, equivalently, walks (read from right to left in figure 12) from height $m$ to $n$, with steps $\leq 1$ and weights

- 1 per step $i \rightarrow i+1$ (no connected subtree)

- $W_{i, j}$ per step $i \rightarrow j$ with $j \leq i$

- $\tilde{g}_{k}$ per walk of length $k-1$ (corresponding to a $k$-valent black face).

For well-labeled mobiles these equations are only valid for strictly positive vertex labels and non-negative flag labels with the boundary conditions

$$
R_{i}=L_{i}=0, \quad \text { for } i \leq 0, \quad W_{i, j}=B_{i, j}=0, \quad \text { for } i<0 \text { or } j<0 .
$$

With these boundary conditions, the set of relations (3.1), (3.2), (3.4) and (3.5) form a closed set determining order by order in the $g$ 's and $\tilde{g}$ 's all the generating functions involved.

As before, the function $R_{n}-R_{n-1}$ generates well-labeled mobiles with root corner labeled $n$, and with at least one label 1. Mapwise, this is nothing but the generating function for Eulerian planar maps with an origin vertex and a distinguished oriented edge $n-1 \rightarrow n$. Moreover, for $m \geq n$, the function $B_{n, m} W_{m, n}-B_{n-1, m-1} W_{m-1, n-1}$ generates the Eulerian planar maps with an origin vertex and a distinguished oriented edge $m \rightarrow n$. In the limit of large labels, we have $R_{i} \rightarrow R, L_{i} \rightarrow L, W_{i, i-m} \rightarrow W_{m}$ and $B_{i, i+m} \rightarrow B_{-m}(m \geq 0)$ and we recover the relations derived in Ref. [4] (up to some rescalings). The function $B_{-m} W_{m}$ is the generating function for Eulerian planar maps with an origin vertex and a distinguished edge $k \rightarrow k-m$ for some $k$.

As before, the function $\log \left(R_{n} / R_{n-1}\right)$ for $n \geq 2$ (resp. $\log R_{1}$ for $n=1$ ) generates Eulerian planar maps with an origin and a distinguished vertex at geodesic distance $n$, weighted by their inverse symmetry factor.

As a final remark, we note that the operators $Q$ and $\tilde{Q}$ of eqns (3.3) and (3.6) are identical to those used in the orthogonal polynomial solution of the two-matrix model 
generating Eulerian maps, respectively implementing the effect of multiplication by an eigenvalue of either matrix. Moreover the recursion relations above are very similar to those governing the genus expansion of this matrix model with the only difference that eq (3.1) should be replaced by $R_{n}=(n / N) /\left(1-L_{n}\right)$ for matrices of size $N \times N$.

\section{Some particular cases of interest}

In this section we show how the construction of Sect. 3 simplifies for two particular classes of planar maps, namely the so-called $p$-constellations [5] and the general planar maps with arbitrary (even or odd) valences [9].

\section{1. -constellations}

A $p$-constellation is an Eulerian planar map such that all black faces have degree $p$ while white faces may have degrees multiples of $p$. Note that the case of maps of even degrees of Sect. 2 corresponds to constellations with $p=2$ by contracting all 2 -valent black faces into single edges.

As before, the vertices of a $p$-constellation with a fixed origin vertex are labeled as in an ordinary Eulerian map by their oriented geodesic distance from the origin. However these labels are more constrained in a p-constellation: the labels along each oriented edge may either increase by 1 or decrease by $p-1$. This is proved by noticing that the length of any two oriented paths from the origin to a given vertex are congruent modulo $p$. Indeed, the difference between the two lengths is equal to the sum of the perimeters of the black faces in between the two paths minus that of the white ones, all of which are multiples of $p$. As a consequence, the labels along oriented edges must increase by 1 modulo $p$, and can neither increase by more than 1 from the geodesic requirement nor decrease by more than $p-1$ because we may use the bypass around the incident $p$-valent black face.

This property results in a drastic simplification of the $p$-mobiles built out of $p$ constellations from the construction of Sect. 3.1. Indeed each black face of a $p$ constellation becomes a univalent black unlabeled vertex in the associated $p$-mobile. This is readily seen by noting that the clockwise sequence of the $p$ labels around a black face is a walk with $(p-1)$ (ascending) steps of +1 and one (descending) step of $-(p-1)$. This unique descending step corresponds to the unique edge incident to the corresponding unlabeled black vertex. This edge carries flags with labels differing by $(p-1)$. This exhausts all flagged edges of the $p$-mobile.

Turning to generating functions, the $p$-constellations are enumerated with a weight $g_{p k}$ per $p k$-valent white face $(k \geq 1)$ and a weight $\tilde{g}_{p}$ per black face. As in Sect. 3.3, the enumeration is performed via the bijection to well-labeled $p$-mobiles. Introducing as before the generating functions $R_{n}$ and $L_{n}$ for rooted $p$-mobiles, and those for halfmobiles $B_{m, n}$ and $W_{m, n}$, the above restrictions imply that

$$
B_{m, n}=\tilde{g}_{p} \delta_{n, m+p-1}
$$


for the trivial half-mobiles whose root vertex is attached to a black univalent vertex. The recursive relations (3.1) and (3.2) remain the same, but with (3.3) replaced by

$$
Q|i\rangle=\tilde{g}_{p}|i+p-1\rangle+R_{i}|i-1\rangle
$$

Equations (2.1), (2.2) and (4.2) form a closed set of equations determining $R_{n}$ order by order in the $g$ 's and $\tilde{g}$ 's, while the $W_{m, n}$ are determined by (3.4).

Note that the black vertices can be removed without loss of information, leading to a slightly simpler definition of $p$-mobiles, now made of only white unlabeled and labeled vertices, with edges connecting only labeled to unlabeled ones, and with the constraint that the clockwise labels around any unlabeled vertex must either decrease by 1 or increase by a quantity of the form $k(p-1)-1, k=1,2,3, \ldots$

The above discussion includes the particular case $p=2$ of bipartite planar maps, and the simplified definition of 2-mobiles matches exactly that of the mobiles of Sect. 2. Moreover, upon taking $\tilde{g}_{2}=1$, the above equations directly reproduce eqns. (2.1), (2.2) and (2.3).

\subsection{Maps with arbitrary valences}

The case of arbitrary planar maps with prescribed face valences may be seen as yet another particular case of Eulerian planar maps, by imposing the condition that all the black faces have valence 2 . These faces may indeed be contracted into regular edges of the ordinary planar map. As a result, these edges may be traversed in both directions; hence we now deal with an unoriented geodesic distance.

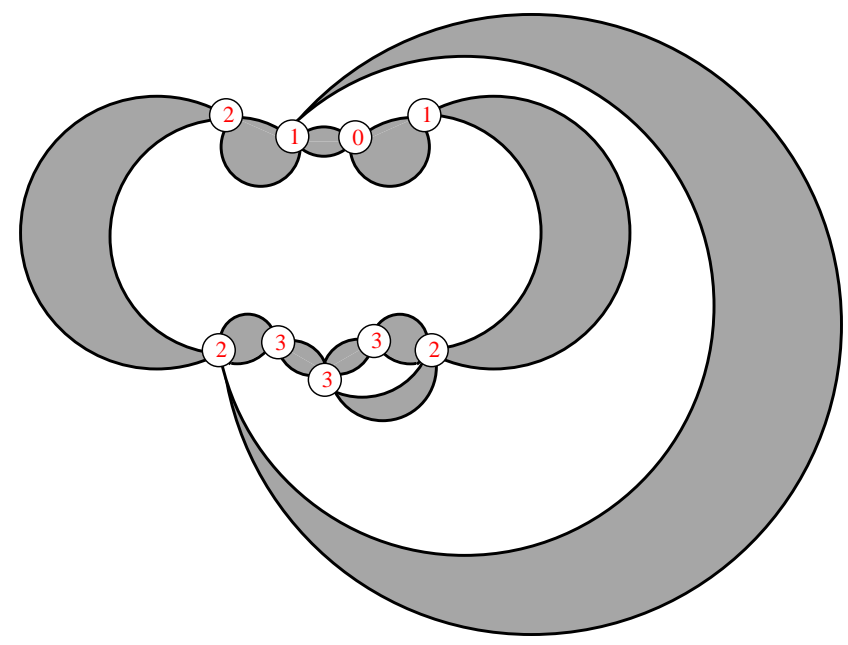

Fig. 15: A sample planar map with arbitrary valences and an origin. The edges are inflated into two-valent black faces producing an Eulerian planar map with only two-valent black faces. As usual, vertices are labeled by their geodesic distance from the origin. The orientation of the edges becomes irrelevant. 

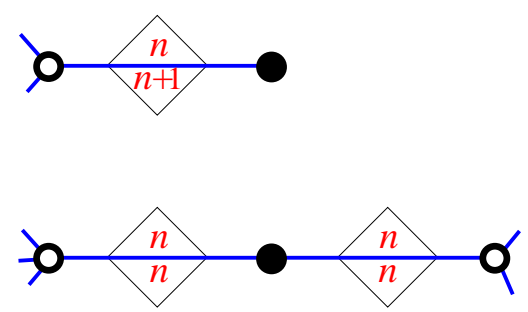
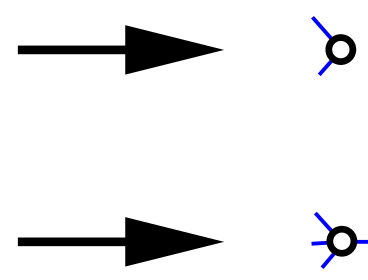

Fig. 16: The two possible situations around an unlabeled black vertex associated with a two-valent black face in an Eulerian map. The arrows indicate simplification rules for the mobiles associated to planar maps with arbitrary valences.

Again this restricts the label configurations: the labels along each oriented edge may differ by $+1,0$ or -1 (see figure 15).

On the associated mobile, we have only two types of unlabeled black vertices as shown in figure 16:

- univalent black vertices, with an incident flagged edge, labeled $n, n+1$

- bivalent black vertices, with two incident flagged edges, both labeled $n, n$.

Turning to the generating functions, we immediately obtain, in addition to eqs. (2.1) and (3.2)

$$
B_{n, n+1}=1, \quad B_{n, n}=W_{n, n},
$$

where we simply have restricted ourselves to $\tilde{g}_{k}=\delta_{k, 2}$, while

$$
W_{n, m}=\sum_{k} g_{k}\left\langle n\left|Q^{k-1}\right| m\right\rangle, \quad m=n, n+1,
$$

where the operator $Q$ reduces to

$$
Q|i\rangle=|i+1\rangle+W_{i, i}|i\rangle+R_{i}|i-1\rangle .
$$
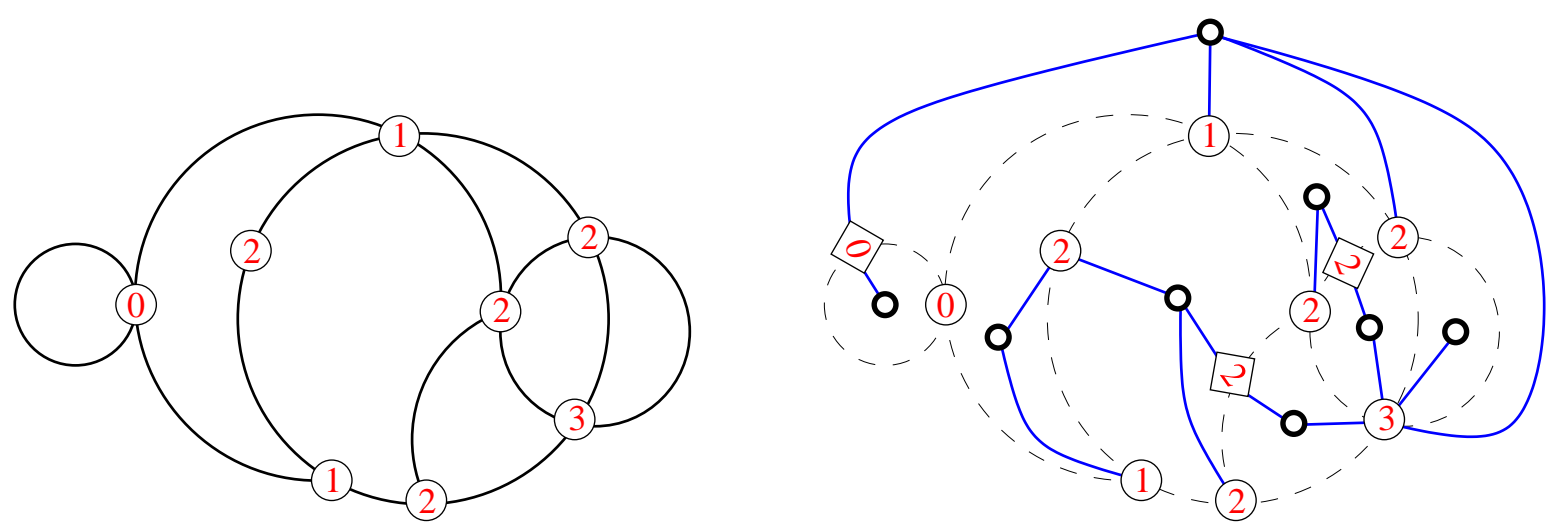

Fig. 17: A sample planar map with arbitrary valences and an origin together with its associated (simplified) mobile. 
The corresponding mobiles may be simplified without loss of information by erasing all univalent black vertices and replacing the bivalent ones and their two incident flagged edges (with, say, labels $n$ ) by a new type of edge, carrying a unique flag (labeled $n$ ), as illustrated in figure 16. The simplified mobiles therefore have only white unlabeled and labeled vertices with two types of edges: unflagged ones connecting labeled to unlabeled vertices, and flagged ones connecting unlabeled vertices to one another. The labels obey the following rule clockwise around unlabeled vertices: a vertex labeled $n$ is followed by a vertex or flag labeled at least $n-1$, while a flag labeled $n$ is followed by a vertex or flag labeled at least $n$. Denoting by $S_{n}=W_{n, n}$ the generating function for half-mobiles with an incident edge flagged $n$, we get the system of equations

$$
\begin{aligned}
R_{n} & =\frac{1}{1-\sum_{k \geq 1} g_{k}\left\langle n\left|Q^{k-1}\right| n-1\right\rangle} \\
S_{n} & =\sum_{k \geq 1} g_{k}\left\langle n\left|Q^{k-1}\right| n\right\rangle \\
Q|i\rangle & =|i+1\rangle+S_{i}|i\rangle+R_{i}|i-1\rangle .
\end{aligned}
$$

Again, this determines $R_{n}$ and $S_{n}$ completely order by order in the $g$ 's. As before, the function $R_{n}-R_{n-1}$ generates arbitrary planar maps with an origin and a distinguished edge $(n-1, n)$, while $S_{n}^{2}-S_{n-1}^{2}$ generates arbitrary planar maps with an origin and a distinguished edge $(n, n)$.

\section{Conclusion}

In this paper, we have established a general bijection between Eulerian planar maps with an origin vertex and so-called mobiles, namely plane trees with specific decorations. The latter carry in particular the information of the oriented geodesic distance from the origin on the original map, and allow for an easy enumeration of maps with prescribed face valences and various distinguished vertices or edges at fixed geodesic distance from the origin. We conclude by discussing some interesting applications.

Local environment in infinite maps:

The bijection gives access to the statistics of the local environment of a vertex (the set of vertices geodesically close to that vertex) in large Eulerian planar maps, by extracting the singular parts of the various generating functions involved (see Ref. [8] for a general scheme and examples). For instance we may extract from the singular part of $R_{n}-R_{n-1}$ the average number of edges at geodesic distance $n$ (i.e. of the form $(n-1, n))$ from a fixed origin in infinite, say, quadrangulations, i.e. bipartite planar maps with only tetravalent faces, and obtain the equation:

$$
\left\langle e_{n-1, n}\right\rangle=\frac{6}{35} \frac{\left(n^{2}+2 n-1\right)\left(5 n^{4}+20 n^{3}+27 n^{2}+14 n+4\right)}{n(n+1)(n+2)} .
$$


Similarly, we may also extract from the singular part of $\log \left(R_{n} / R_{n-1}\right)$ the average number of vertices at geodesic distance $n$ from an origin in infinite quadrangulations, with the result

$$
\left\langle v_{n}\right\rangle=\frac{3}{35}\left((n+1)\left(5 n^{2}+10 n+2\right)+\delta_{n, 1}\right) .
$$

Integrability properties:

The recursion relations obtained in this paper match those found in Ref. [6] through the bijection with blossom trees, but now with a different (dual) interpretation in terms of mobiles. Many of these equations were found to be integrable (i.e. have a maximal number of independent integrals of motion) and have exact solutions as given in [6], which directly translate into compact expressions for the generating functions of rooted mobiles, as well as for the corresponding rooted maps.

Branching processes:

The mobiles introduced in this paper are (decorated) labeled trees. It is tempting to interpret them as spatially branching processes, by viewing the tree as coding the genealogy of a population while the labels code positions of its members on the integer line. Such an interpretation was exploited in Ref. [7] to relate the generating function $R_{n}$ for planar quadrangulations to the escape probability of a species from a domain of the integer line. It would be interesting to see whether the rules for labels in mobiles have a natural translation in terms of population evolution, and to study their scaling limit around multicritical points.

Acknowledgments: All the authors acknowledge the support of the EU network on "Discrete Random Geometry", grant HPRN-CT-1999-00161.

\section{References}

[1] D. Arquès, Les hypercartes planaires sont des arbres très bien étiquetés, Discr. Math. 58(1) (1986) 11-24.

[2] E. Bender and E. Canfield, The number of degree-restricted rooted maps on the sphere, SIAM J. Discrete Math. 7(1) (1994) 9-15.

[3] D. Boulatov and V. Kazakov, The Ising model on a random planar lattice: the structure of the phase transition and the exact critical exponents, Phys. Lett. B186 (1987) 379-384.

[4] M. Bousquet-Mélou and G. Schaeffer, The degree distribution in bipartite planar maps: application to the Ising model, arXiv:math.CO/0211070.

[5] M. Bousquet-Mélou and G. Schaeffer, Enumeration of planar constellations, Adv. in Applied Math., 24 (2000) 337-368.

[6] J. Bouttier, P. Di Francesco and E. Guitter, Geodesic distance in planar graphs, Nucl. Phys. B663[FS] (2003) 535-567, arXiv:cond-mat/0303272. 
[7] J. Bouttier, P. Di Francesco and E. Guitter, Random trees between two walls: Exact partition function, J. Phys. A: Math. Gen. 36 (2003) 12349-12366, arXiv:condmat/0306602.

[8] J. Bouttier, P. Di Francesco and E. Guitter, Statistics of planar maps viewed from a vertex: a study via labeled trees, Nucl. Phys. B675[FS] (2003) 631-660, arXiv:condmat/0307606.

[9] J. Bouttier, P. Di Francesco and E. Guitter, Census of planar maps: from the one-matrix model solution to a combinatorial proof, Nucl. Phys. B645[PM] (2002) 477-499, arXiv:cond-mat/0207682.

[10] E. Brézin, C. Itzykson, G. Parisi and J.-B. Zuber, Planar Diagrams, Comm. Math. Phys. 59 (1978) 35-51.

[11] P. Chassaing and B. Durhuus, Statistical Hausdorff dimension of labelled trees and quadrangulations, arXiv:math.PR/0311532.

[12] P. Chassaing and G. Schaeffer, Random Planar Lattices and Integrated SuperBrownian Excursion, Probability Theory and Related Fields 128(2) (2004) 161-212, arXiv:math.CO/0205226.

[13] R. Cori and B. Vauquelin, Planar maps are well labeled trees, Canad. J. Math. 33 (5) (1981) 1023-1042.

[14] M. Douglas, The two-matrix model, in Random Surfaces and Quantum Gravity, O. Alvarez, E. Marinari and P. Windey eds., NATO ASI Series B: Physics Vol. 262 (1990).

[15] A. Hurwitz, Über Riemann'sche Flächen mit gegebenen Verzweignungspunkten, Math. Ann. 39 (1891) 1-60.

[16] J. F. Marckert and A. Mokkadem, Limit of normalized quadrangulations: the Brownian map, arXiv:math.PR/0403398.

[17] G. Schaeffer, Conjugaison d'arbres et cartes combinatoires aléatoires, PhD Thesis,

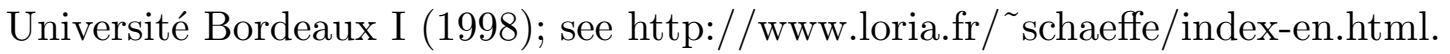

[18] G. Schaeffer, Bijective census and random generation of Eulerian planar maps, Electronic Journal of Combinatorics, vol. 4 (1997) R20.

[19] W. Tutte, A Census of Planar Maps, Canad. J. of Math. 15 (1963) 249-271.

[20] W. Tutte, A Census of slicings, Canad. J. of Math. 14 (1962) 708-722. 